\title{
Comprehensive characteristics analysis of Low Carbon - Environmental Protection Power Generation
}

\author{
Xiao Zhanhui ${ }^{1}$, Liu Dunnan ${ }^{2}$, Zhou Long ${ }^{1}$, Wang Qiang ${ }^{2}$ \\ ${ }^{1}$ Guangdong Power Grid Corporation, Guangzhou City 510600, P.R. China \\ ${ }^{2}$ North China Electric Power University, Beijing 102206, P.R. China
}

\begin{abstract}
The first step to study environmental problems caused by power generation is focusing on electrical energy and carbon emission coordination scheduling and classifying the generating units by near zero emission, ordinary fossil fuels and carbon capture. And then make an in-depth analysis on electrical energy and SOx, NOx and smoke based on coordination scheduling. Eventually form a multi-objective coordination framework between electrical energy and various pollutants after the comprehensive analysis of their coordination scheduling under the environmental constraints.
\end{abstract}

\section{Introduction}

Highlighting energy conservation and emission reduction, The Chinese Government regards reducing energy consumption per unit GDP by $20 \%$ as a rigid index during the period of “11th five-year plan”. China tried out energy-saving power generation dispatching to promote the electric power industry, mainly industry consuming primary energy and discharging pollution, carrying out energy conservation and emission reduction since the end of 2007.

There are a large number of literatures about the power generation scheduling. Ref [1-3] shows a energysaving generation scheduling method based on all kinds of improved algorithm. Ref [4-6] presents an optimization model of energy-saving generation scheduling for two goals of economic operation and environmental protection. The research framework of low-carbon electricity technology is built after analyzing the situation and characteristics of low-carbon electricity in ref 7. The priliminary low carbon power dispatching decision-making model is set up and the electrical carbon scheduling characteristics of different types of power is described in ref 8 . The importance of energy-saving generation scheduling to reduce the $\mathrm{CO} 2$ emission and the relationship between energy-saving generation scheduling and low-carbon electricity scheduling are showed in ref 9.

The CO2 emission should be taken seriously as well as generation scheduling in the electric power dispatching trading. Therefore, there is no doubt that electrical and carbon scheduling trading characteristics is the foundation of low carbon economy.

\section{Comprehensive energy efficiency index system}

In the low carbon power dispatching trading, the goal is to realize the maximization of resource utilization efficiency. The principle is make the most effective resources priority to produce electricity. Therefore, when arranging power plan, low carbon, energy saving, emission, economic marginal cost (power generation), all kinds of pollutants in the process of the electric power production and management cost should be considered. There are four performances to evaluate power generation benefits.

1) Carbon emission performance. Carbon emission mainly refers to the carbon dioxide emission in the process of generating.

2) Energy consumption performance. Electricity energy consumption refers to the fuel energy consumption in the process of power generation.

3) Pollutant emission performance. Pollutants mainly refers to various types of generating units discharge sulfur dioxide (SO2), nitrogen oxide (NOx) and soot emissions in the process of generating.

4) A feed-in tariff performance. A feed-in tariff refers to various types of units of electricity price. It means the cost for power grid.

According to the analysis of the performances, the index system frame of comprehensive energy efficiency of generating units is shown as figure 1 .

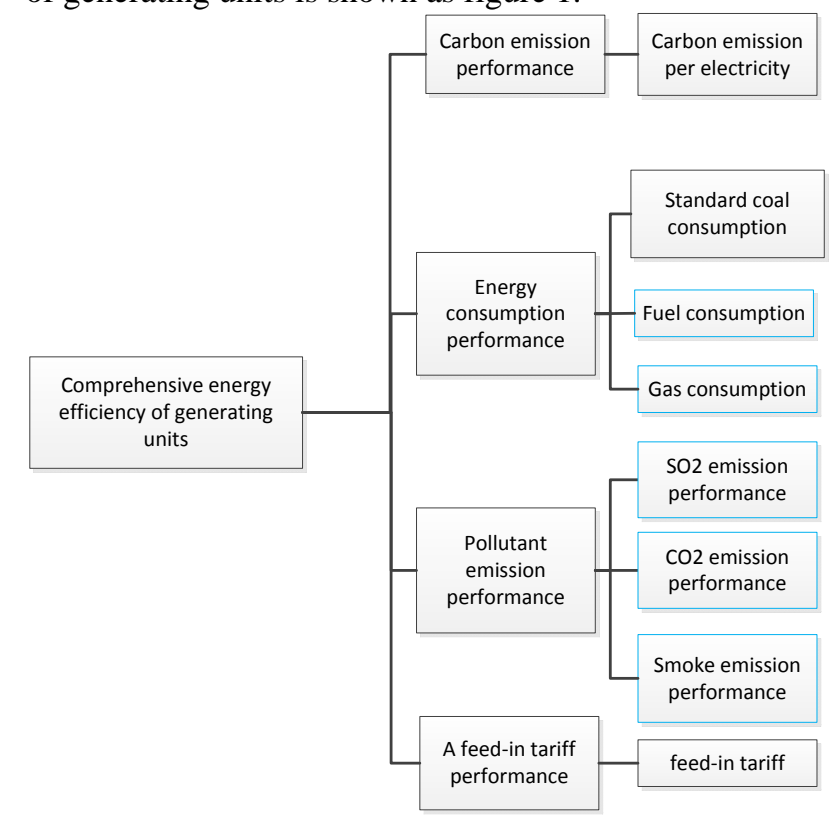

Figure 1 Comprehensive energy efficiency index system 


\section{Electricity - carbon scheduling transaction analysis}

Define generated energy g as the independent variable and corresponding $\mathrm{CO} 2$ emission $\mathrm{E}$ as the dependent variable. $E=f(g)$, the function relationship between $g$ and $\mathrm{E}$, reflects the electrical and carbon scheduling trading characteristics. Electrical carbon characteristic function have three types according different types power.

\subsection{Near zero emission power}

Near zero emission power means renewable energy sources such as hydropower, nuclear power, wind power and photovoltaic power which discharge negligible $\mathrm{CO} 2$. The function can be expressed as:

$$
E=f(g)=0
$$

\subsection{Ordinary fossil fuel power}

Ordinary fossil fuel power means thermal power plant which generating by coal, oil and natural gas. This fuels produce $\mathrm{CO} 2$ when get fired to generate and discharge it to the atmosphere. The function of $\mathrm{CO} 2$ emission can be expressed as:

$$
E=F \cdot f
$$

$F$ means the fuel quantity of consumed in the process of combustion. $f$ means the amount of $\mathrm{CO} 2$ emission which uint fuel gets burned adequately. The function of generated energy can be expressed as:

$$
g=F \cdot q \cdot \eta
$$

$q$ means calorific value of unit fuel. $\eta$ is the generating efficiency.

United function (2) and (3), the electrical carbon characteristic function of this power can be expressed as:

$$
E=\frac{f}{q} \cdot \frac{1}{\eta} \cdot g
$$

The relationship of "generating- carbon emission" for ordinary fossil fuel power in scheduling can be expressed by the functions above. For other power plants, their electrical carbon characteristic functions are different with their different fuels and power technology. Generating efficiency usually waves in a small range with the change of the generating level. But generating efficiency remains a certain constant in this mode just for simple.

\subsection{Carbon capture plant}

Carbon capture plant means traditional coal-fired power plant with an individual carbon capture system which can separate CO2 from the emission and convey it to the safety storage site so that it can isolate from the atmosphere in the long term. In other words, carbon capture system reduces the $\mathrm{CO} 2$ emission equivalently.

It will reduce the external power output of power plant because capturing CO2 needs much energy. Define $\alpha$ as the loss energy for capturing unit of CO2 and $E_{c}$ as the total quantity of captured $\mathrm{CO} 2$. And then the equivalent emission of plant $E$ 'can be expressed as:

$$
E^{\prime}=E-E_{c}=F \cdot f-E_{c}
$$

The external power output of carbon capture plant $g^{\prime}$ can be expressed as:

$$
g^{\prime}=g-\alpha \cdot E_{c}=F \cdot q \cdot \eta-\alpha \cdot E_{c}
$$

United function (5) and (6), the electrical carbon characteristic function of carbon capture plant can be expressed as:

$$
E^{\prime}=\frac{f}{q \cdot \eta} \cdot g^{\prime}+\left(\frac{f \cdot \alpha}{q \cdot \eta}-1\right) \cdot E_{c}
$$

In the formula (7), $E_{c}$ equals 0 if plant fails to capture $\mathrm{CO} 2$. And then formula (7) is simplified as formula (4). $E_{c}$ takes the maximum $E_{c}^{\max }$ when plant capture all $\mathrm{CO} 2$, that is:

$$
\left\{\begin{array}{l}
0 \leq E_{c} \leq E_{c}^{\max } \\
E_{c}^{\max }=\gamma \cdot E \\
g^{\min } \leq g \leq g^{\max }
\end{array}\right.
$$

Substitute formula (4) into formula (8), than is:

$$
E_{c}^{\max }=\gamma \cdot \frac{f}{q} \cdot \frac{1}{\eta} \cdot g
$$

$g^{\text {min }}$ and $g^{\text {max }}$ mean the minimum and maximum of generating output. $\gamma$ means the capture rate of carbon capture plant and its value is usually between $80 \% \sim 80 \%$.

Compared formula (4) and (7), the relationship of generating and carbon emission is not match anymore. Carbon capture plant can change the emission of $\mathrm{CO} 2$ by adjusting the value of $E_{c}$ at the fixed $g$ so that the plant can run in different conditions. All of conditions form its operation range.

\section{The scheduling transaction of electricity and other emission}

\subsection{The analysis of electrical and sulfur scheduling trading characteristics}

In low carbon economy oriented electric power dispatching trading. Electrical and sulfur scheduling trading means scheduling trading considering $\mathrm{SO} 2$ emission. There is no doubt that electrical and sulfur scheduling trading characteristics is the foundation of low carbon economy oriented electric power dispatching trading.

Define generated energy g as the independent variable and corresponding SO2 emission En as the dependent variable. $E s=f(g)$, the function relationship between $g$ and Es, reflects the electrical and sulfur scheduling trading characteristics. 
It will greatly affect Es whether unit install the denitration device which can improve the SO2 emission characteristics.

The desulfurization of electricity price will increase 0.015 yuan per kilowatt hour.

\subsection{The analysis of electrical and nitrate scheduling trading characteristics}

In low carbon economy oriented electric power dispatching trading. Electrical and nitrate scheduling trading means scheduling trading considering $\mathrm{NOx}$ emission. There is no doubt that electrical and nitrate scheduling trading characteristics is the foundation of low carbon economy oriented electric power dispatching trading.

Define generated energy $g$ as the independent variable and corresponding NOx emission En as the dependent variable. $E n=f(g)$, the function relationship between $g$ and En, reflects the electrical and nitrate scheduling trading characteristics.

It will greatly affect En whether unit install the denitration device which can improve the NOx emission characteristics.

The denitration of electricity price increased 0.008 yuan per kilowatt hour since January 2013.

\subsection{The analysis of electrical and smoke scheduling trading characteristics}

Electrical and smoke scheduling trading means considering the emission of smoke when scheduling trading under the environmental constraints.

\section{The analysis of environmental scheduling trading characteristics}

Scheduling trading under the environmental constraints should consider the emission of $\mathrm{CO} 2, \mathrm{SO} 2$, NOX, and smoke. There is no doubt that "electricalCO2-SO2-NOX-smoke” scheduling trading characteristics is the foundation of low carbon economy oriented electric power dispatching trading.

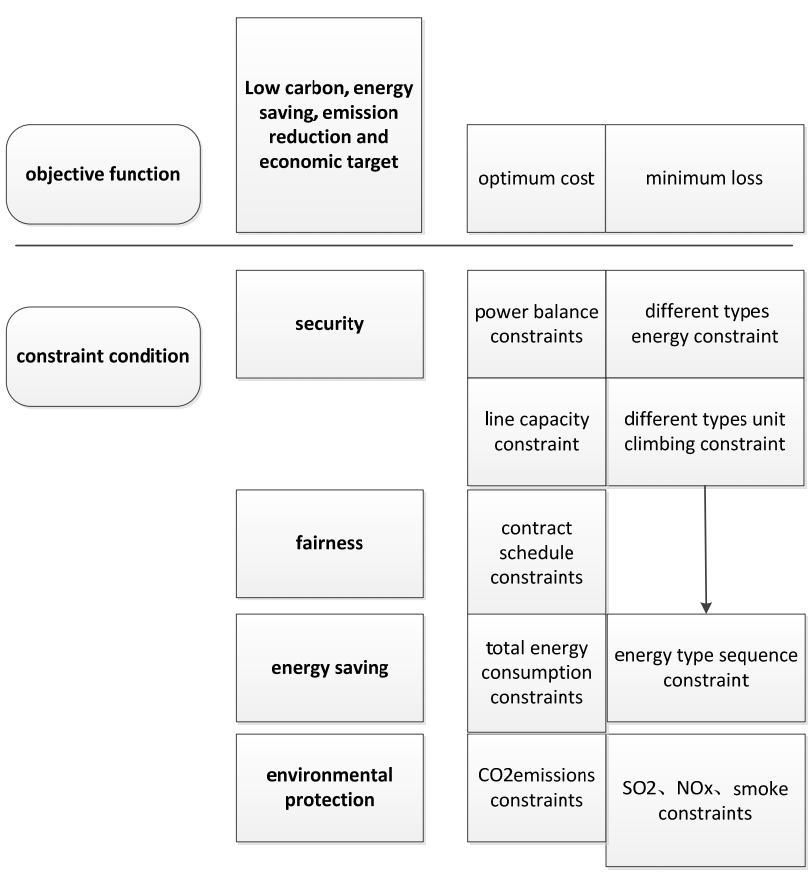

Figure 2 The multi-objective coordination framework of "electrical-CO2-SO2-NOX-smoke”

The multi-objective coordination framework of “electrical-CO2-SO2-NOX-smoke” is shown as figure 2.

(1)The target of objective function is to minimize the cost of power generation and the loss of power grid;

(2)The constraints condition shows the goal of safety, justice, energy saving, emission reduction and low carbon;

(3)Safety constraints include power balance constraints, line capacity constraints, different generators output constraints and climbing constraints;

(4)Justice constraints include contract schedule constraints;

(5)Energy saving constraints include energy consumption constraints and generating sequence constraint;

(6)Environmental constraints mean $\mathrm{CO} 2, \mathrm{SO} 2$, NOX, and smoke constraints.

\section{Conclusion}

Different energy types have different unit power emission of "carbon, sulfur, nitrate, smoke" in actual power generation.

The higher the proportion of thermal power unit in the grid, the more negative indexes of "carbon, sulfur, nitrate, smoke", because the emission of thermal power unit is obviously huge.

Gas thermal power unit only discharges $1 / 3$ of CO2 and 1/5 of NOx than fossil-fired unit. And gas thermal power unit almost discharge negligible $\mathrm{SO} 2$ and smoke.

Biomass energy is renewable energy but it will discharge $\mathrm{CO} 2$ and smoke and face the same environmental problem as fossil-fired unit.

Other renewable energy and new energy units are positive in the target of "carbon, sulfur, nitrate, smoke".

In conclusion, from the macroscopic view, the propotion of different energy resouces in energy structure 
has a strong affect to "carbon, sulfur, nitrate, smoke"; From the long term, it is the foundation way that lowering the propotion of thermal power to realize the scheduling transaction of "carbon, sulfur, nitrate, smoke".

\section{References}

[1] Liu Jing , Luo Xianjue . Environmental economic dispatching adopting multiobjective random blackhole particle swarm optimization algorithm [J]. Proceedings of the CSEE, 2010, 30(34): 105-111.

[2] Zhao Weixing, Lin Cheng, Sun Bin, et al. Study on economic dispatch method of the optimal composite coal loss under security constraints $[\mathrm{J}]$. Power Systems Protection and Control, 2010, 38 (9): 18-22.

[3] Su Peng, Liu Tianqi, Zhao Guobo, et al. An improved particle swarm optimization based multiobjective load dispatch under energy conservation dispatching[J]. Power System Technology, 2009, 33(5): 48-53(in Chinese).

[4] Yu Jie, Li Yang, Xia Anbang. Distributed optimization of generation dispatch schedule considering environmental protection and economic profits [J]. Proceedings of the CSEE, 2009, 29(16): 63-68(in Chinese).

[5] Zhang Xiaohua, Zhao Jinquan, Chen Xingying. Multi-objective unit commitment fuzzy modeling and optimization for energy-saving and emission reduction [J]. Procee-dings of the CSEE, 2010, 30(22): 71-76(in Chinese).

[6] Fan Yuhong, Zhang Wei, Ye Yongsong, et al. Energy conservation generation dispatching mode in regional power network based on high-low matching of coal consumption rates of units $[\mathrm{J}]$. Power System Technology, 2009, 33 (6): 78-81(in Chinese).

[7] Kang Chongqing, Chen Qixin, Xia Qing. Prospects of low-carbon electricity [J]. Power System Technology, 2009, 33(2): 1-7(in Chinese).

[8] Chen Qixin, Kang Chongqing, Xia Qing. Mechanism and modeling approach to low-carbon power dispatch $[\mathrm{J}]$. Automation of Electric Power Systems, 2010, 34(12): 18-22(in Chinese).

[9] Chen Qixin, Zhou Tianrui, Kang Chongqing, et al. An assessment model of low-carbon effect and its application to energy saving based generation dispatching[J]. Automation of Electric Power Systems, 2009, 33(16): 18-22(in Chinese). 